\section{Enttäuschende Phase-III-Studie mit ASA404}

\section{Die Kombination der gefäßzerstörenden Substanz (Vascular Disrupting Agent, VDA) ASA404 mit Carboplatin und Paclitaxel hatte in einer Phase-II- Studie beim fortgeschrittenen nichtkleinzelligen Lungenkarzinom (NSCLC) erstaunliche Erfolge erzielt. Jetzt liegt die Phase-III-Studie vor.}

Z u den neueren Anti-Tumor-Strategien gehört der Versuch, Blutgefäße des Tumors mit Hilfe von VDA zu unterbrechen und so dessen Versorgung abzuschneiden. Zu den bisher am besten untersuchten VDA gehört ASA404 (Vadimezan), ein Flavonessigsäure-Analogon. Es löst Apoptose in den endothelialen Zellen von Tumorgefäßen aus, das genaue molekulare Ziel ist jedoch unbekannt. In einer randomisierten Phase-II-Studie führte die Kombination aus ASA404, Carboplatin und Paclitaxel bei NSCLC-Patienten im Vergleich zur Chemotherapie allein zu einem um neun Prozentpunkte verbesserten Ansprechen (31 vs. $22 \%$ ) und einem um fünf Monate verlängerten Gesamtüberleben (14,0 vs. 8,8 Monate).

Die Ergebnisse der nun vorliegenden Phase-III-Studie mit NSCLC-Patienten im Stadium IIIB oder IV wurden deshalb mit Spannung erwartet. Mehr als 200 Zentren in 20 Ländern haben daran teilgenommen. Alle 1.299 Patienten erhielten $200 \mathrm{mg} / \mathrm{m}^{2}$ Paclitaxel sowie Carboplatin (AUC 6,0), nur jeder zweite Proband bekam alle drei Wochen $1.800 \mathrm{mg} / \mathrm{m}^{2}$ ASA404 intravenös. Auf sechs Chemotherapie-Zyklen folge eine ASA404-Erhaltungstherapie oder ein Placebo. Primärer Endpunkt war das Gesamtüberleben, sekundäre Endpunkte unter anderem Gesamtansprechrate (ORR) und progressionfreies Überleben (PFS).

Nach der Interimsanalyse wurde die Studie gestoppt. Das Gesamtüberleben zwischen ASA404- und Placebo-Arm unterschied sich nicht (13,4 vs. 12,7 Monate; Hazard Ratio [HR] 1,01). Auch in Subgruppenanalysen (nach Geschlecht bzw. histologischem Typ) zeigten sich keine Überlebensunterschiede. Das mediane PFS lag bei 5,5 Monaten in beiden Armen (HR 1,04), die Gesamtansprechrate bei jeweils $25 \%$.

Die Toxizität war insgesamt vergleichbar. Nur Grad-4-Neutropenie und Schmerzen an der Infusionsstelle waren in der ASA404-Gruppe deutlich häufiger ( 27 vs. $19 \%$ bzw. 10 vs. $0,5 \%$ ).
Fazit: Die Zugabe von ASA404 zu Carboplatin und Paclitaxel wurde gut vertragen. Sie konnte aber die Wirksamkeit der Erstlinientherapie beim fortgeschrittenen NSCLC nicht verbessern. Aufgrund des milden Nebenwirkungsprofils stellt sich die Frage, ob ASA404 ausreichend dosiert war.

Christina Berndt

Lara PN et al. Randomized phase III placebocontrolled trial of carboplatin and paclitaxel with or without the vascular disrupting agent vadimezan (ASA404) in advanced nonsmall-cell lung cancer. J Clin Oncol. 2011; 29(22):2965-71.

Kommentar von PD Dr. Reinmuth: Vadimezan (ASA404) ist eine niedermolekulare gefäßzerstörende Substanz, die aufgrund direkter Zerstörung von Endothelzellen zu einem Verschluss intratumoraler Blutgefäße und damit zu einem sekundären Untergang von Tumorzellen führen soll. Auch wenn der eigentliche Wirkmechanismus unbekannt ist, unterscheidet er sich prinzipiell von dem antiangiogener Substanzen, die vorwiegend die Neubildung von Gefäßen beeinflussen sollen.

Die Substanz scheint gut vertragen zu werden mit meist nur leicht erhöhter Grad3/4-Neutropenie-Rate in der aktuellen PhaseIII-Studie. Nach vielversprechenden PhaseII-Daten in der Kombination mit Carboplatin und Paclitaxel als Erstlinientherapie [McKeage MJ et al. Br J Cancer. 2008;99:2006-12; McKeage MJ et al. Lung Cancer. 2009;65:1927] wurde die nun publizierte Phase-III-Studie nach einer Interimsanalyse gestoppt. Neben dem primären Endpunkt Gesamtüberleben wurden auch sekundäre Endpunkte wie progressionsfreies Überleben und Ansprechen nicht erreicht. Nachdem eine weitere PhaseIII-Studie (ATTRACT-2) in der Zweitlinie für die Zugabe von Vadimezan zu Docetaxel ebenfalls keinen Vorteil zeigte, wurde die weitere klinische Entwicklung des Medikaments eingestellt.

Wie kommen diese ernüchternden Ergebnisse nach vielversprechend erscheinender Überlebensverlängerung von 8,8 auf 14,4 Monate aus einer ähnlich dosierten Phase-
II-Studie zustande [McKeage MJ et al. 2008]? Mehrere Gründe können angeführt werden:

Auffällig ist das gute Abschneiden der Patienten im Kontroll-Arm mit einem medianen Gesamtüberleben von 12,7 Monaten, das deutlich höher liegt als in der Phase-II-Studie (8,8 Monate) oder anderen Phase-III-Studien wie z. B. ECOG-4599 (10,3 Monate) [Sandler A et al. N Engl J Med. 2006;355:2542-50]. Ein stärkerer Einschluss asiatischer Patienten und vermehrte weitere Chemotherapie könnten zu dem guten Ergebnis beigetragen haben. Andererseits beleuchten die aktuellen Daten auch die Ergebnisse aus der Phase-II-Studie neu: In dieser Studie waren nur 73 Patienten eingeschlossen und der große Unterschied des Überlebensvorteils war nicht signifikant ( $p=0,33$; Hazard Ratio 0,73; 95\%-Konfidenzintervall 0,39-1,38). Daher verdeutlicht die aktuelle Phase-III-Studie die Variabilität falsch positiver und möglicherweise auch falsch negativer Phase-II-Daten und die Schwierigkeit, Ergebnisse aus (zu) kleinen Phase-IIStudien zu verallgemeinern.

Ein weiteres, möglicherweise entscheidendes Problem ist die geringe präklinische Entwicklung der Substanz: Der Wirkmechanismus ist nicht geklärt, mögliche prädiktive Marker für eine Präselektion von Patienten

\section{„Das Potenzial der Vascular Disrupting Agents kann derzeit gar nicht richtig beurteilt werden."}

fehlen. Unklar ist eine mögliche Beeinträchtigung der Effektivität von ASA404 durch die steroidhaltige Komedikation. Zudem wurde in der Studie eine Erhaltungstherapie mit Vadimezan oder Placebo bis zur Krankheitsprogression durchgeführt, wobei der Nutzen nicht belegt und die Hypothese unklar ist.

Es liegt nun an der präklinischen Forschung, diese Fragen zu klären und neue hypothetische Ansätze zu finden. Das Potenzial der Vascular Disrupting Agents kann daher derzeit gar nicht richtig beurteilt werden. Es wäre schade, wenn eine zu frühe klinische Testung an einer unselektierten Patientenpopulation die weitere Entwicklung dieser Substanzklasse verhindert.

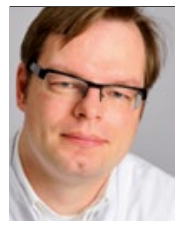

PD Dr. Niels Reinmuth

Onkologie, Krankenhaus Großhansdorf n.reinmuth@ kh-grosshansdorf.de 